\title{
FPGA Implementation of Fault Tolerant Full Adder Design for High Speed VLSI Architectures
}

\author{
Somashekhar, Vikas Maheshwari, R. P. Singh
}

\begin{abstract}
The essential goal is to distinguish and diminish the deficiencies in full Adder configuration making use of Self checking and Self Repairing Adder Block. The tempo of chip disappointment is straightforwardly relative to chip thickness. A framework should be flaw tolerant to diminish the frustration rate. The nearness of different troubles can demolish the usefulness of complete snake. This paper displays a region proficient flaw tolerant complete snake shape that may repair issues without interfering with the everyday assignment of a framework. The combo and duplicate is finished through way of making use of Xilinx ISE 14.7 and actualized on FPGA Spartan three..
\end{abstract}

Keywords: VLSI, Fault Tolerance, full Adder, Self-Checking, Self Repairing, FPGA Spartan 3, Verilog, Xilinx ISE 14.7.

\section{INTRODUCTION}

Issue tolerant plans are explicitly applied for sort of responsibilities such defend device, satellite and guarantee measures and severa others. a mix-u.s.a.go off in a framework may cost some harm or have an effect on human presence moreover. anyhow, some systems are reconfigurable, which repair it. these device can't be spoil and fasten that mistake precisely. inside the cutting-edge situation, VLSI circuits emerge as extra noteworthy complicated for the reason that the CMOS trademark duration is scaling in nanometre machine. This downscaling makes the circuit more and more smaller and touchy to the brief deficiencies. brief deficiency go off inside the integrated beams, cross-communicate and electricity deliver commotion. what is extra, innovation scaling comparatively will build the odds of the nearness of eternal flaw. The concept of self-checking and deficiency tolerant is delivered to conform to the inconvenience of shortcoming. Self-checking shows the recognition of deficiencies and disregards the overhead connected with trouble healing.

the extensive majority of oneself checking bureaucracy re-execute the guidance for the deficiency healing. besides this way diminishes the exhibition of the machine due to the fact that each single damaged hub are resurfaced. be that as it could, this method does by no means again make certain the

Revised Manuscript Received on July 10, 2019.

Somashekhar, Research Scholar, Dept. of ECE, SSSUTMS, Sehore (M.P), India(Email: somashekhar49@ gmail.com)

Dr.Vikas Maheshwari, Associate Professor, Dept. of ECE, Bharat Institute of Engineering and Technology, Hyderabad. India.(Email: maheshwarivikas1982@gmail.com)

Dr.R. P.Singh, Vice-Chancelor \& Professor, Dept. of ECE, SSSUTMS, Sehore (M.P), India.(Email: provc@sssutms.co.in) circuit as a result of electromagnetic clamors, astronomical

lack reclamation if the problem is perpetual. Viper performs an implication of uses in virtual machine and has high-quality significance DSP activities. There are styles of issues manifest in complete viper shape as an example single difficulty and twofold trouble. single deficiency makes great one yield broken right away. but, twofold deficiency makes every the yields insufficient without delay. it's far difficult to locate and connect the twofold flaw. This makes the layout additional complex and requires more outstanding place overheads. It makes the problem tolerant complete snake design a be checked of perfect importance. in the beyond, severa tactics are brought to shape oneself checking total viper utilising gadget excess or time repetition. those strategies expand as an achievement in figuring out the difficulty besides bombs in demonstrating the precise locale of that shortcoming. alongside those traces, it makes the opposite module defective in mild of the engendering of the deliver. on this paper, we support a sparkling out of the plastic new shortcoming tolerant layout which demonstrates the single and twofold difficulty with its and programmed issue solving is in like manner possible in this structure.

Stepped forward microelectronic innovation have approved forefront advanced structures to develop to be extra prominent helpless to shortcomings. it has been found that the issue of unmarried-event disenchanted in advanced frameworks has turn out to be greater excellent with the developing unpredictability of gadget on a chip, alongside bringing clock cycles right down to boom excessive jogging recurrence. The design of a minimal circuit on a chip is tremendous in expressions of clamor but makes special inconveniences as a ways as unwavering fine. Scientists concur that rebate in system size will development equipment failures in predetermination processors. warm biking, dielectric behavior and biasing of advanced incorporated circuits have additionally expedited numerous transient and eternal flaws. with a view to deal with the above-refered to troubles, the mind of self-checking and version to internal failure had been offered. A framework may be flaw quiet in the event that it remains unaffected through strategies for a deficiency or within the event that it suggests a shortcoming as fast since it occurs. A framework may act naturally looking at at the off danger that it delivers a non-coded yield in response to each created trouble. A contraption may be really self-checking (TSC) if it's miles every shortcoming 
comfortable and self-checking out. The concept of TSC is used in particular bundles, including self-recuperation systems, self-checking math cause devices (ALUs), and so on.

Self-checking by means of and massive underlines the invention of deficiencies and disregards the overhead related with shortcoming reclamation. restriction of oneself checking strategies require re-execution of directions for deficiency healing. be that as it is able to, the re-execution approach consequences system execution since all sports associated right now or circuitously to the deficient module have to be re-achieved. furthermore, re-execution can't make certain trouble restoration, especially if there may be a perpetual problem or put on-out difficulty. consequently, on line discovery and self-fixing is needed in a actually dependable machine engineering. In virtual gadget plans, the viper has a wide fashion of bundles. within the beyond, severa strategies have been pursued to give self-checking in snake circuits both by using utilizing equipment or time touchy repetition.

those methodologies can run over a blunders with out showing its licensed place in view of flaw engendering due to bring. on this paper, we suggest every other self-checking and self-solving full snake in which the wrong total viper module is probably distinguished. Self-checking and reestablish have been done the usage of the determined connection amongst Sum and convey out. The Sum and skip on out bits of a total viper is probably identical to each outstanding even as every one of the three information resources are equal. The Sum and bring-out bits will be supplemented while any of the three resources of data are unmistakable. The proposed self-solving snake is suit for fixing a few shortcoming, relying at the viper size.

\section{PREVIOUS WORK}

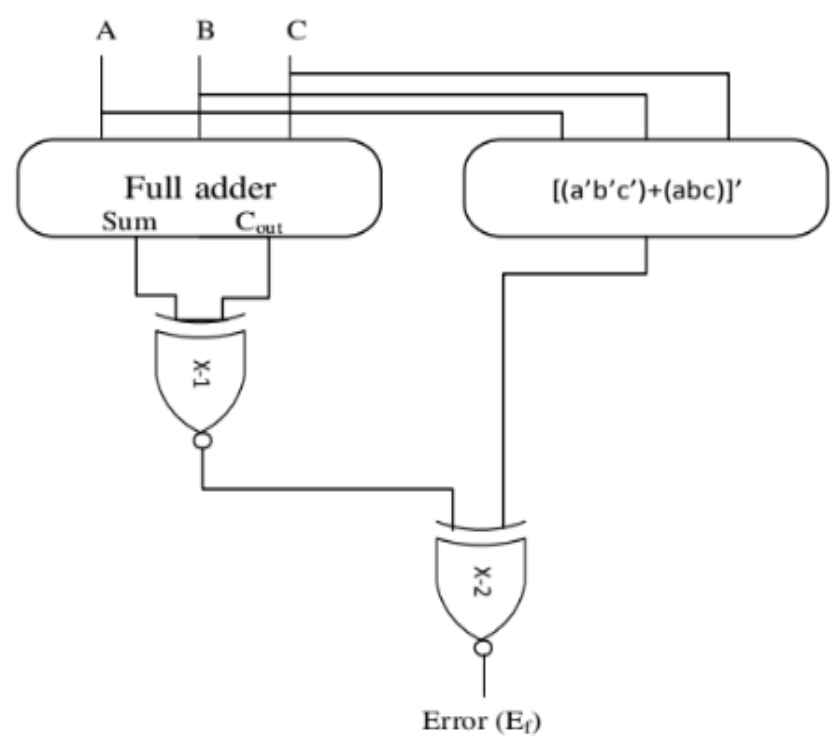

Figure 1. Self Checking full adder

Self repairing adder removes the problem of fault propagation thru convey took place in the preceding self-checking design by using the use of indicating the correct place of fault. The design of self-checking whole adder is tested in determine 1 . The hardware requirements of self-checking adder are complete adder cell, equal tester and two XOR gates for self-attempting out the fault.

Sum $=\mathrm{A}^{\wedge} \mathrm{B}^{\wedge} \mathrm{Cin}$

Cout $=A B+\operatorname{Cin}(A+B)$

$\mathrm{Eqt}=\sim(($ A. B .Cin $)+($ ABCin $))$

The XOR entryway (X-1) is implemented for contrasting the combination and bring yields produced via the whole snake cell. It takes a shot at the rule that entirety and convey yields may be equal even as all of the data associated are equal and the entire and produce

yields can be complement to every different even as any of the three statistics resources related is precise when it comes to residual facts resources. The XOR door $(\mathrm{X}-2)$ is implemented to don't forget the yields of XOR entryway $(\mathrm{X}-1)$ and utilitarian unit $\left[\left(\mathrm{A}^{\prime} \mathrm{B}^{\prime} \mathrm{C}^{\prime}\right)+(\mathrm{ABC})\right]^{\prime}$. The flaw is spoken to as Ef. on the off danger that Ef is 0, it demonstrates that there's a shortcoming and if Ef is 1 it demonstrates the hassle unfastened circumstance.

The primary issue of this form is that it fizzles, if twofold deficiency occurs in every whole and bring yields straight away. For this case, Ef demonstrates the shortcoming unfastened situation and flawed yield spread to the following unit thru convey and cause them to faulty.

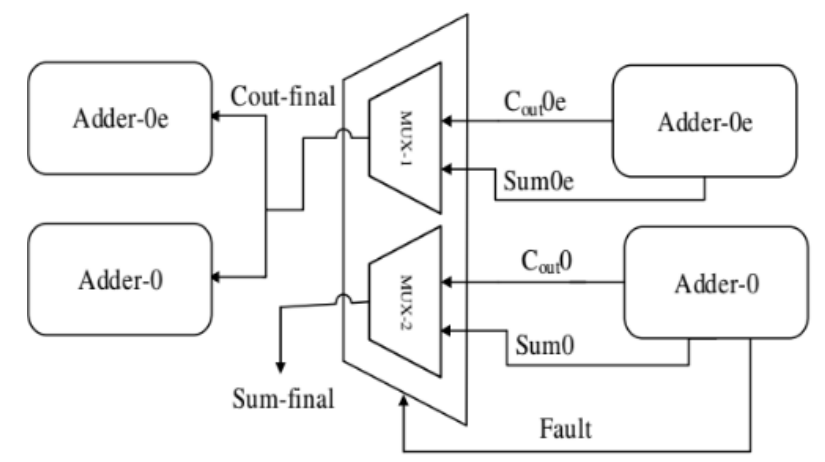

Figure 2 . Self Repairing Adder.

The flaws recognized in self checking approach are fixed with the helpful asset of changing the defective complete snake portable with some other repetitive whole viper as appeared in figure 2 . The working conduct is that one snake is sketches as a normal viper while the another viper is craftsmanship as repetitive snake. The equipment necessities of this design are self-checking whole adders and two multiplexers. The drawback of this arrangement is that it falls flat while twofold blames ascend at once. In this model, the issue sign yield (Ef) of this plan recommends that there can be no shortcoming and self-fixing configuration will now not fine art on this in statute. in this way, issue isn't fixed by utilizing oneself fixing viper and complete snake proposes the damaged yield. 


\section{PROPOSED WORK}

Proposed Self Checking Full Adder

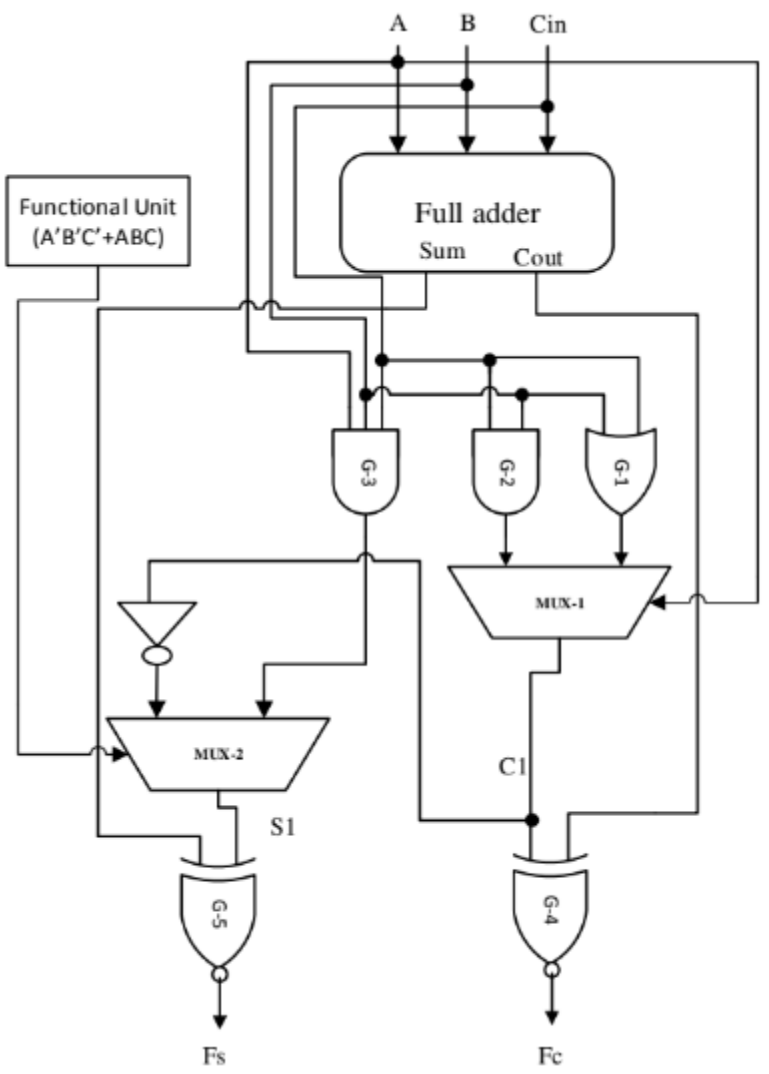

Figure 3 . Proposed Self Checking Full Adder Design

The output expressions for the sum and carry outputs of the full adder is shown in equations 1 and 2.

$$
\text { Sum }=\mathrm{A} \oplus \mathrm{B} \oplus \mathrm{Cin}
$$

Cout $=\mathrm{AB}+\mathrm{BCin}+\mathrm{Cin} \mathrm{A}$

Table.1. Truth Table of Self checking full adder design

\begin{tabular}{|c|c|c|c|c|c|c|c|c|c|c|}
\hline $\mathrm{A}$ & $\mathrm{B}$ & $\mathrm{C}$ & $\mathrm{S}$ & $\mathrm{c}$ & $\mathrm{G}$ & $\mathrm{G}$ & $\mathrm{G}$ & $\mathrm{E}$ & $\mathrm{Fc}$ & $\mathrm{Fs}$ \\
$\mathrm{u}$ & $\mathrm{y}$ & 1 & $\mathrm{~m}$ & 3 & $\mathrm{~g}$ & & \\
\hline 0 & 0 & 0 & 0 & 0 & 0 & 0 & 0 & 1 & 0 & 1 \\
\hline 0 & 0 & 1 & 1 & 0 & 1 & 0 & 0 & 0 & 1 & 0 \\
\hline 0 & 1 & 0 & 1 & 0 & 1 & 0 & 0 & 0 & 1 & 0 \\
\hline 0 & 1 & 1 & 0 & 1 & 1 & 1 & 0 & 0 & 1 & 1 \\
\hline 1 & 0 & 0 & 1 & 0 & 0 & 0 & 0 & 0 & 1 & 0 \\
\hline 1 & 0 & 1 & 0 & 1 & 1 & 0 & 0 & 0 & 0 & 1 \\
\hline 1 & 1 & 0 & 0 & 1 & 1 & 0 & 0 & 0 & 0 & 1 \\
\hline 1 & 1 & 1 & 1 & 1 & 1 & 1 & 1 & 1 & 1 & 0 \\
\hline
\end{tabular}

The proposed self-checking complete snake is based upon the main given beneath.

1. the full yield is opposite to the bypass on yield when vectors of facts sources $\mathrm{A}, \mathrm{B}$ and $\mathrm{C}$ aren't ascend to as an example $(010,100)$. It exhibits that beside $(000)$ and (111) input blends of $\mathrm{A}, \mathrm{B}$ and $\mathrm{C}$ sum yield is contrary to the pass on yields as showed up in desk 1 .

2.the full yield is proportionate to the skip on yield whilst data vectors A, B and C are comparable for example $(000$, 111). It exhibits that for introductory (000) and remaining (111) enter mixes of A, B and C, total yield is comparable to the pass on yields as showed up in desk 1 .

The proposed self-checking full snake is based upon the principle given beneath.

1 . The combination yield is contrary to the bypass on yield while vectors of wellsprings of information $\mathrm{A}, \mathrm{B}$ and $\mathrm{C}$ are not ascend to as an instance (010,one hundred). It indicates that beside (000) and (111) input mixes of A, B and C sum yield is backwards to the pass on yields as confirmed up in desk 1.

2.The mixture yield is proportional to the pass on yield when facts vectors A, B and C are identical for example (000, 111). It suggests that for introductory (000) and closing (111) input blends of

A, B and C, combination yield is proportional to the skip on yields as showed up in desk 1 .

\section{PROPOSED SELF REPAIRING FULL ADDER LAYOUT}

The proposed self-fixing full snake requires unimportant region overhead than the prevailing plans. The motion of the proposed shape is accomplished the use of multiplexers suffering from $\mathrm{Fs}$ and $\mathrm{Fc}$. The yield $\mathrm{Fs}$ and $\mathrm{Fc}$ are made by using the proposed self-checking complete snake. The proposed self-fixing association does not require any live via snake cellular which are used to override the faulty snake as used inside the beyond self-fixing complete snake. in this system, defects are constant through using inverter in place of the hold full snake mobile as confirmed up in Fig. five.

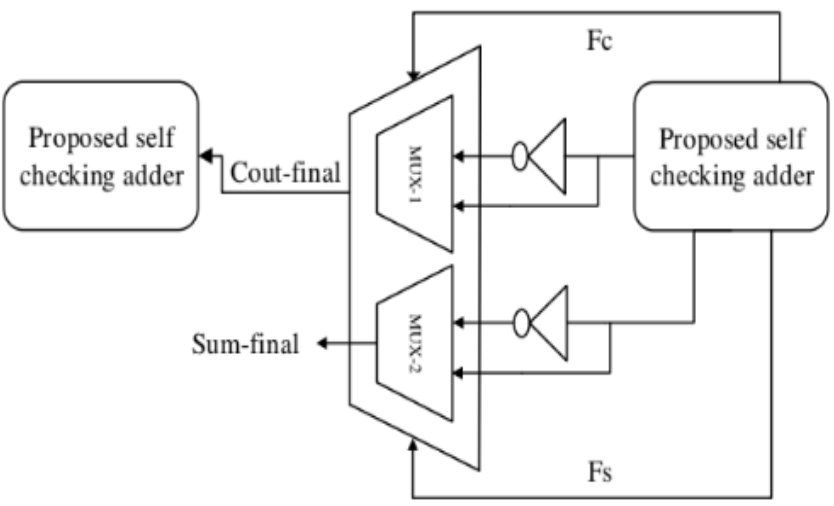

Figure 4.Proposed self-repairing full adder design 
The project of the proposed plan depends at the manipulate signals ( $\mathrm{Fs}$ and $\mathrm{Fc}$ ) given through oneself checking full viper. at the off chance that the manage sign Fs is 0 , it demonstrates

that there is no shortcoming inside the complete yield and the mixture yield originating from the whole viper mobile may be selected via the multiplexer to create the last all out. Multiplexers are sorted out the usage of transmission passages. in any case, If the administer sign is Fs 1, it shows that there is a burden inside the blend yield. The imperfect whole yield starting from the full snake cell is typical through using the inverter. The steamed total yield is other than picked through the multiplexer to make irrefutably the last aggregate. additionally, if the regulate sign Fc is zero, it suggests that there is no issue inside the bring yield, and the pass on yield beginning from the entire snake convenient might be chosen by strategy for the multiplexer to deliver the last bring. obviously, If the manage sign is Fc 1, it demonstrates that pass on yield is imperfect. The insufficient pass on yield is fixed by strategy for using the inverter. The changed pass on yield is similarly chosen by using the multiplexer to make irrefutably the last pass on. on along these lines, the flawed snake adaptable is fixed and changed over into the weakness segregated snake. along these lines, this framework can restore single and twofold deficiency occurs on the total and pass on yields to the detriment of

\section{SIMULATION \& SYNTHESIS RESULTS}

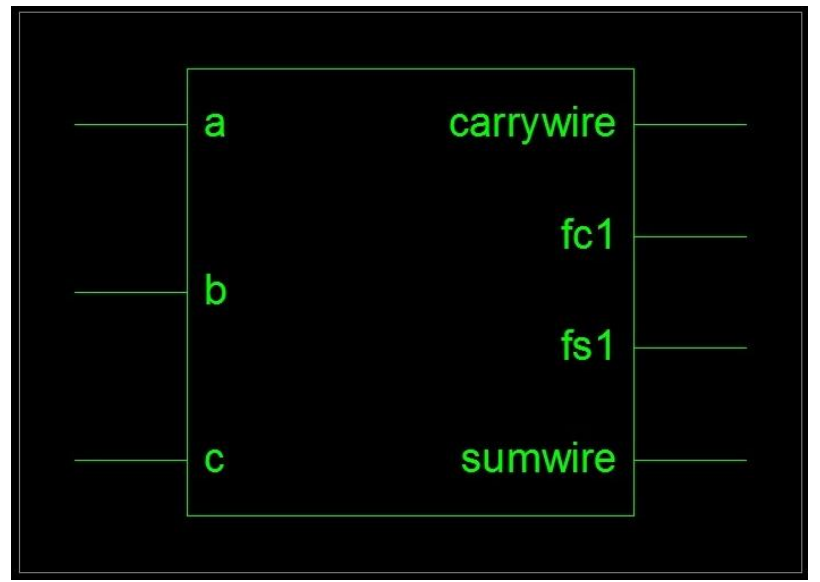

Figure 5. Top module of self checking Full Adder

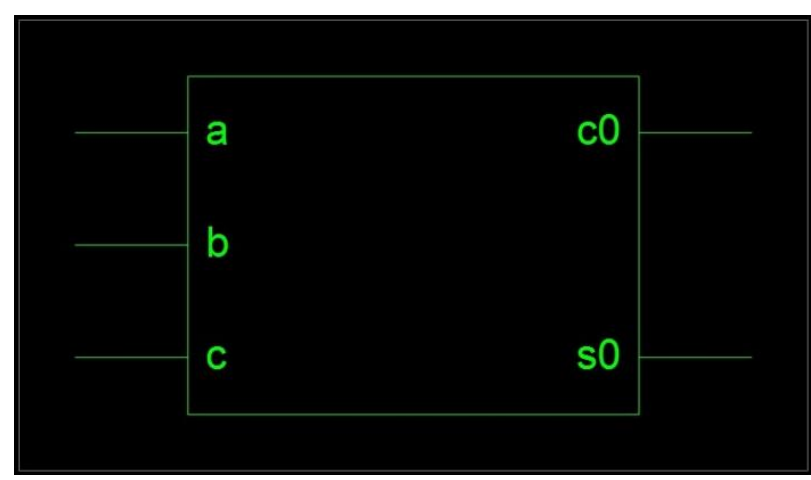

Figure 6. Top module of self repairing Full Adder immaterial hardware.

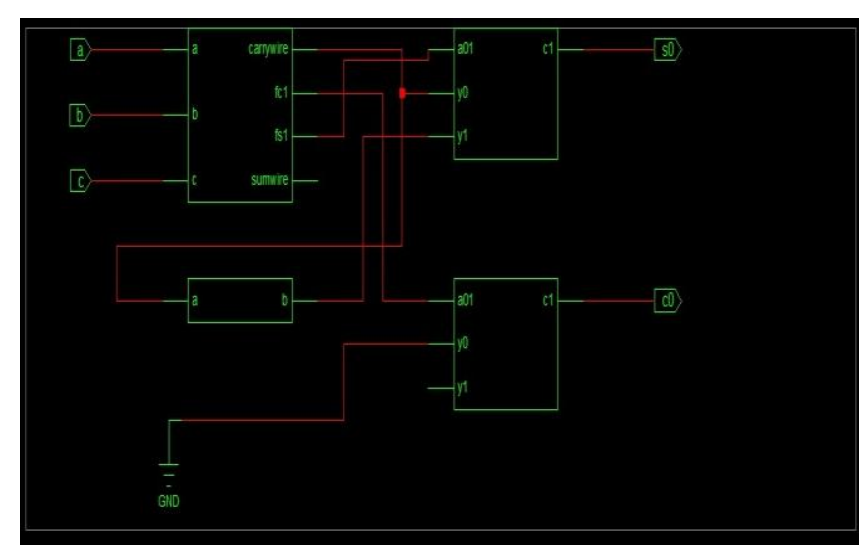

Figure 7. RTL Schematic Self checking Full Adder

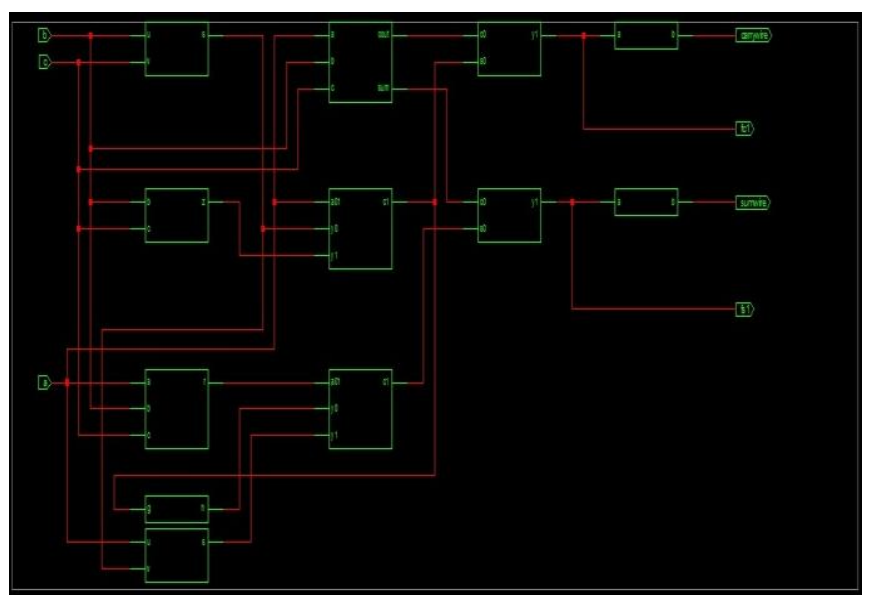

Figure 8. RTL Schematic of self repairing Full Adder

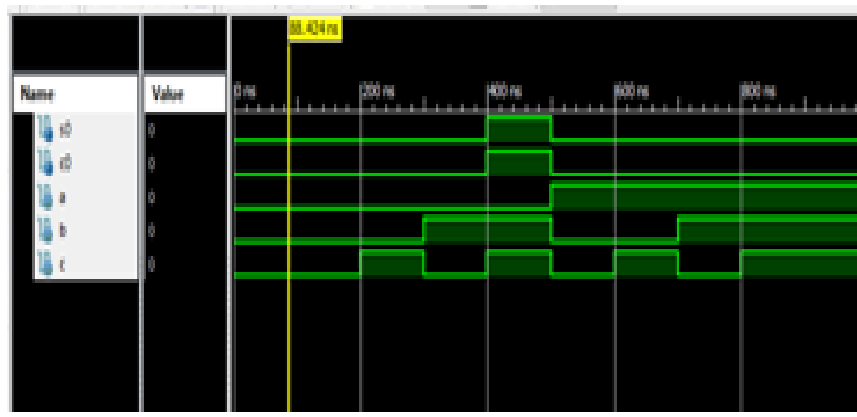

Figure 9. Simulation result of Self Checking Full Adder

Here, 3 inputs $\mathrm{A}, \mathrm{B}, \mathrm{C}$ are taken. Inputs are $\mathrm{A}=0, \mathrm{~B}=0$, $\mathrm{C}=0$. We get the Output as $\mathrm{Fc}=1 \& \mathrm{Fs}=1$.

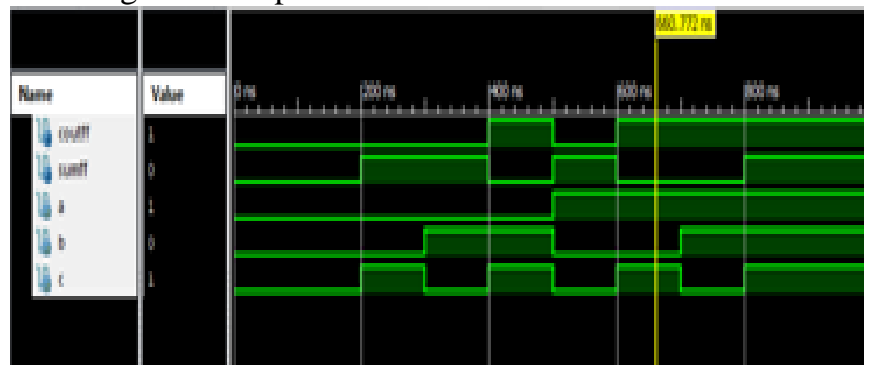

Figure 10. Simulation results of Self repairing

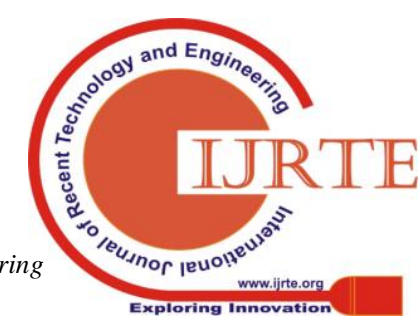


Here $A, B, C$ are the inputs taken.Here, the operation is based on the control signals $F$ and $F c$.Inputs are $A=1, B=0$, $\mathrm{C}=1$. The Outputs are coutff $=1 \&$ sumff $=0$.

Delay Analysis of Self Repairing:

Cell: in->out fanout Delay Delay Logical Name (Net Name)

IBUF: I->O

LUT3:I0->O

$1 \quad 1.2180 .595$ c_IBUF (c_IBUF)

20.7040 .447 c01 (c0_OBUF)

OBUF: I->O

3.272

s0_OBUF (s0)

Total

$6.236 \mathrm{~ns}(5.194 \mathrm{~ns}$ logic, $1.042 \mathrm{~ns}$ route $)$

$(83.3 \%$ logic, $16.7 \%$ route $)$

\section{IMPLEMENTATION RESULTS}

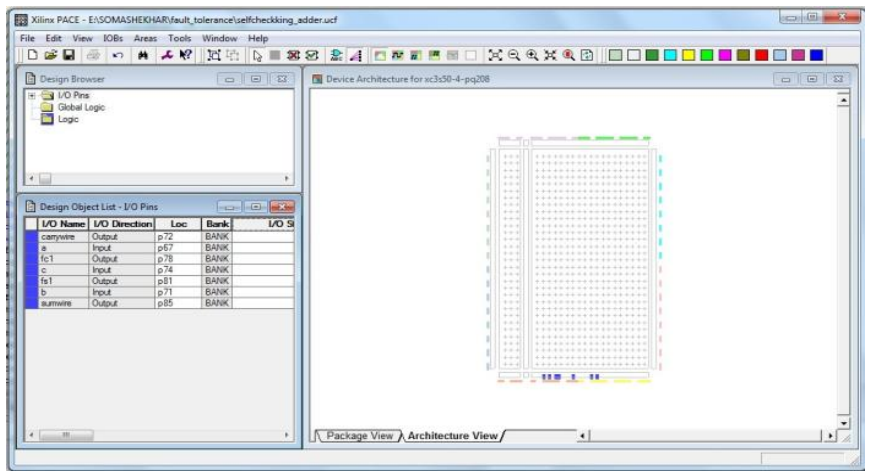

Figure 11. UCF file generation

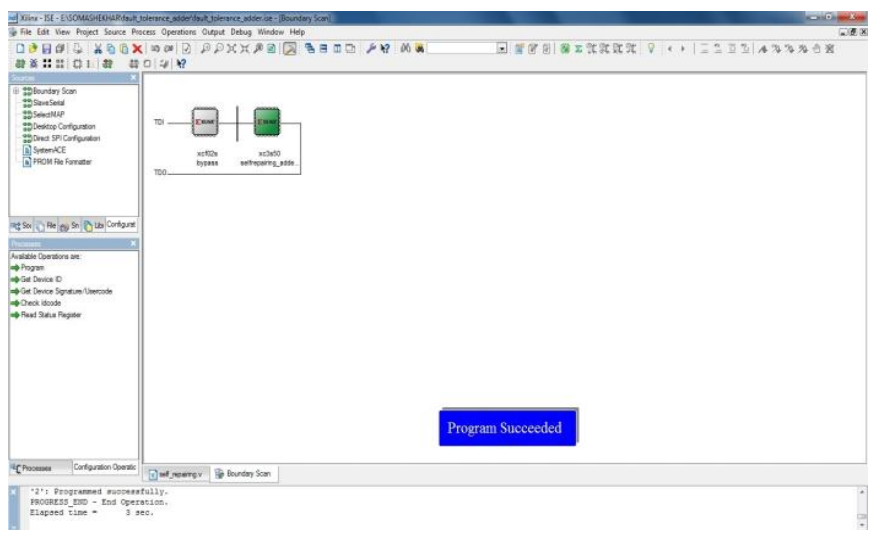

Figure 12. Boundary Scan checking

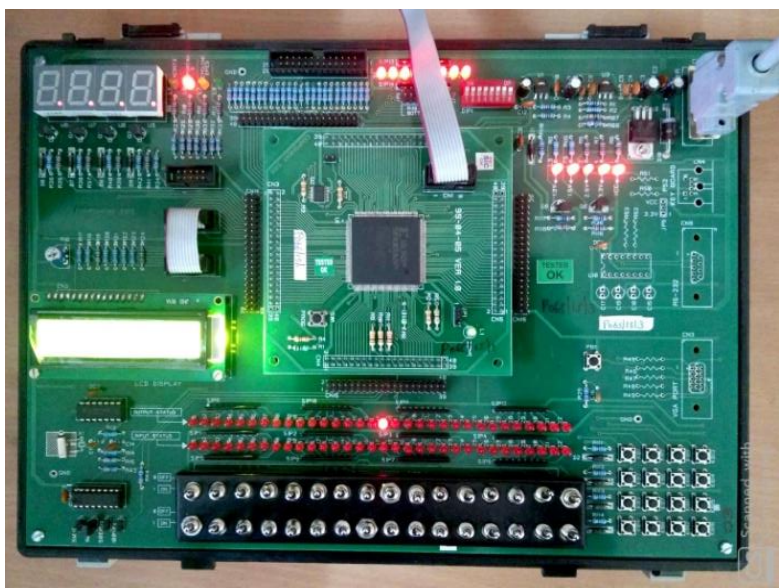

Figure 13. FPGA Implemented results

\section{CONCLUSION}

In this arrangement, a total snake is provided pointing the flaw identification and remedy usefulness. The proposed arrangement eat up lesser spot because of the reality rather than changing the deficient viper with excess snake, damaged entirety and produce yield is altered utilizing an inverter and multiplexer. subsequently, proposed issue tolerant organization has extreme speed (6.236ns) and lesser area than oneself fixing whole viper.

\section{REFERENCES}

1. Biernat, J., "fast issue tolerant adders," worldwide magazine crucial pc-based structures, Vol. 1, Nos. half of/three, 2010, pp.117-127.

2. A. Mukherjee and A. S. Dhar, "plan of a Self-Reconfigurable Adder for Fault-Tolerant VLSI engineering," 2012 worldwide Symposium on electronic device design (ISED), Kolkata, 2012, pp. 90 - 96 . doi: 10.1109/ISED.2012.21.

3. Somashekhar, Vikas Maheshwari, R. P. Singh, "assessment of Micro Inversion to embellish Fault Tolerance in extreme beat VLSI Circuits", global examinations mag of Engineering and innovation (IRJET), amount: 06 inconvenience: 03, web page 5041-5044, Mar 2019.

4. W.- T. Cheng and J. H. Patel, "A negligible check set for numerous flaw discovery in swell convey adders," IEEE Trans. Comput., vol. C-36, no.7, July 1987, pp. 891-895.

5. S. gupta, A. Jasuja and R. shandilya, "constant shortcoming tolerant whole viper the utilization of deficiency restriction," 2018 IEEE global understudies' show on electric, Electronics and workstation innovative data (SCEECS), Bhopal, 2018, pp. 1-6.doi: 10.1109/SCEECS.2018.8546908.

6. Somashekhar Malipatil, R. Basavaraju and Praveen kumar Nartam. "Low power and high pace pass on pick Adder design utilizing Verilog" IOSR diary of VLSI and sign Processing Vol. 6 Iss. 6 (2016) p. seventy seven - 80 one ISSN: $2319-4197$.

7. Akbar, Muhammad Ali, and Jeong-A. Lee. "Self-fixing viper utilizing deficiency limitation." Microelectronics Reliability fifty four, no. 6 (2014): 1443-1451.

8. Somashekhar Malipatil, Chandragiri Bhanu prakash, N Dasharath, "FPGA Implementation of over the top speed convey pick Adder format the utilization of Spartan", IOSR diary of VLSI and sign Processing (IOSR-JVSP) degree 9, issue 2, Ser. I (Mar. - Apr. 2019), PP 01-05.

9. Bejagam Divya, Alekhya Bonkuri, Madhavi Bandi, Somashekhar Malipatil, "format and confirmation of low power and unbalanced pace convey select snake utilizing Verilog", worldwide magazine of imaginative research mind (IJCRT), ISSN:2320-2882, quantity.6, issue 1, page No pp.1786 - 1790, March 2018 